\title{
Lipid Simulations: A Perspective on Lipids in Action
}

\author{
Ilpo Vattulainen ${ }^{1,2,3}$ and Tomasz $\operatorname{Rog}^{1}$ \\ ${ }^{1}$ Department of Physics, Tampere University of Technology, Fl-33101 Tampere, Finland \\ ${ }^{2}$ Department of Applied Physics, Aalto University School of Science and Technology, Fl-00076 Aalto, Finland \\ ${ }^{3}$ MEMPHYS —Centre for Biomembrane Physics, University of Southern Denmark, Odense, Denmark \\ Correspondence: Ilpo.Vattulainen@tut.fi
}

In this article, we provide an overview of lipid simulations, describing how a computer can be used as a laboratory for lipid research. We briefly discuss the methodology of lipid simulations followed by a number of topical applications that show the benefit of computer modeling for complementing experiments. In particular, we show examples of cases in which simulations have made predictions of novel phenomena that have later been confirmed by experimental studies. Overall, the applications discussed in this article focus on the most recent state of the art and aim to provide a perspective of where the field of lipid simulations stands at the moment.

ipids are very diverse in their structures and Lfunctions (Sackmann 1995; Mouritsen 2005; van Meer 2005). They are a crucial component of numerous biological entities such as membranes, lipoprotein particles, and lipid droplets, and they are involved in numerous cellular functions related to, for example, signaling and energy storage. Importantly, as lipids also compartmentalize biological membranes by creating membrane domains with different physical properties, lipids also affect or even govern membrane proteins and their functionality (McIntosh and Simon 2006; Lingwood and Simons 2010).

Although experiments are the cornerstone of lipid research, they are limited in resolution, permitting one to unravel biological phenomena only to a limited extent. Especially difficult to deal with are molecular scales with an objective to probe phenomena in the nanometer regime over timescales less than a microsecond. Molecular simulations, on the other hand, have no such limits with regard to resolution. Validated simulation models can be used to consider all sorts of phenomena, ranging from selectivity of ion channels to interactions of lipids with membrane proteins, and further to nonequilibrium lipid trafficking and domain as well as pore formation (Bjelkmar 2009; Bucher et al. 2010; Fan et al. 2010a,b).

The first simulations of lipid systems were performed in the early 1980s (Kox et al. 1980; van der Ploeg and Berendsen 1982, 1983). Starting from those times when solvent-free membranes composed of 32 lipids were simulated for about 80 picoseconds (van der Ploeg and

Editor: Kai Simons

Additional Perspectives on The Biology of Lipids available at www.cshperspectives.org

Copyright (C) 2011 Cold Spring Harbor Laboratory Press; all rights reserved; doi: 10.1101/cshperspect.a004655

Cite this article as Cold Spring Harb Perspect Biol 2011;3:a004655 
Berendsen 1982), the field of lipid simulations has matured to a stage in which the scales simulated in atomistic detail cover tens of nanometers (about $10^{5}-10^{6}$ atoms) and several microseconds (Bjelkmar 2009; Dror 2009). This progress in atomistic simulations has been supported by the development of coarse-grained models and multiscale simulation techniques able to elucidate phenomena over scales much larger than the molecular ones (Ayton and Voth 2009; Murtola et al. 2009). The currently used particlebased coarse-grained models are appropriate for studies of systems of millions of particles over timescales of the order of 10-100 microseconds (Reynwar et al. 2007; Apajalahti 2010), and the situation continuously improves. Today, simulations can provide a great deal of insight into a variety of phenomena that are not tractable by experiments. Simulations are no longer used as tools for confirming what has been found in experiments; instead they have predictive power, guiding experiments to focus on novel phenomena. Current aims to bridge molecular simulations with computational systems biology foster the field further, coupling molecular and cellular phenomena to one another.

In this article, we provide an overview of lipid simulations, describing how a computer can be used as a laboratory for lipid research. We briefly discuss the methodology of lipid simulations followed by a number of topical applications that show the benefit of simulations. The applications given here as examples of simulations' role for lipid research focus on the most recent state of the art and aim to provide a perspective of where we stand at the moment. A brief discussion of the prospects of lipid simulations closes this article.

\section{SIMULATION METHODOLOGY IN A NUTSHELL}

Extensive and detailed descriptions of computer simulation techniques as applied to molecular systems are many and available elsewhere (Tieleman et al. 1997; Frenkel and Smit 2002; Praprotnik et al. 2007; Murtola et al. 2009; Senn and Thiel 2009); thus, here we focus only on the essentials. The starting point of every simulation is to decide the questions one wants to clarify, because they determine the relevant length and timescales, and they in turn determine the technique that is most appropriate for a given problem. If the phenomenon one wishes to understand deals with electronic degrees of freedom, such as a chemical reaction because of lipases acting on a lipid, then the method of choice is quantum mechanics. For physical processes in which electronic properties are not important but which take place over molecular scales, the ideal technique is usually classical atomistic molecular dynamics (MD). At scales much larger than the molecular size, atomistic techniques are no longer reasonable, and one has to resort to coarse-grained (CG) models that usually represent the system as coarse-grained particles or with fields.

Here, we focus on two techniques that are most often applied to molecular simulations of lipid systems: atomistic and CG molecular dynamics. In both cases, there are three main steps that need to be accomplished to simulate a lipid system. First, given the molecular structures of lipids, one has to construct the initial configuration of a system one aims to study, such as a hydrated lipid bilayer. Second, to describe intramolecular and intermolecular interactions one needs the Hamiltonian (force field), whose parameters are often determined from quantum-mechanical simulations and experiments. Finally, to generate the evolution of the system in time, one has to integrate Newton's equations of motion for the particle positions and velocities a sufficient number of times.

The heart of molecular modeling is the force field. The interactions are described by a potential energy function typically having the form presented in Figure 1. There are two types of interactions: bonded and nonbonded ones. Bonded interactions include bonding, bending, and dihedral (torsional) terms, whereas nonbonded interactions typically include electrostatic and Lennard-Jones (van der Waals) interactions. The set of these interactions, together with their parameter values, constitute the force field. There are many force fields used 


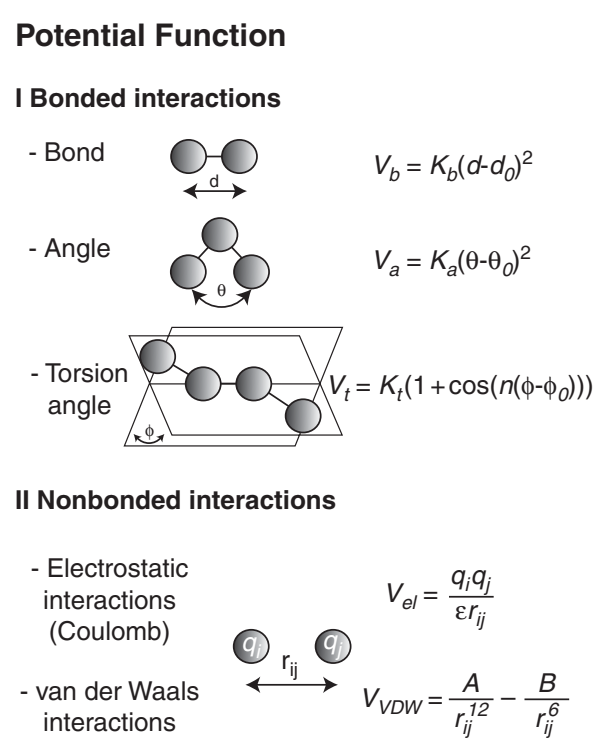

Figure 1. Representation of potential function terms (bond, angle, torsion angle, Coulomb, and van der Waals) often used in classical molecular dynamics simulations and molecular modeling in general. Here, $d$ is bond length; $d_{0}$ its equilibrium value; $\theta$ is angle with $\theta_{0}$ representing its equilibrium value; $\varphi$ is torsion angle; $\varphi_{0}$ is 0 or 180 degrees; and $\mathrm{n}$ represents phase. $K_{b}, K_{a}$, and $K_{t}$ and force constants $q_{i}$ and $\mathrm{q}_{j}$ denote partial charges of atoms that are not covalently bonded, and $\epsilon$ is dielectric constant. $r_{i j}$ is distance between a pair of interacting atoms, and $\mathrm{A}$ and $\mathrm{B}$ are constants that depend on the chemical nature of interacting atoms.

in simulations of biomolecular systems, the most important ones being Amber, Charm, OPLS, and GROMOS (van Gunsteren et al. 2006). These force fields differ in how their parameters are derived, and they often have a different number of terms with different forms in the potential energy function.

For practical purposes, a number of more advanced algorithms are used to complement the above-mentioned three key items. For instance, to minimize artifacts because of a finite system size, one typically employs periodic boundary conditions that effectively allow one to consider a system of infinite size. Further, to model the system under conditions that mimic experimental ones, one often uses thermostats and barostats to maintain the temperature and pressure at desired values.

Although classical MD simulations can provide a great deal of insight into lipid systems, they obviously also have a number of limitations. First, as one is dealing with a classical method, one can only consider physical processes because chemical reactions cannot be investigated without accounting for electronic degrees of freedom. For the same reason, there are currently no effective and commonly accepted methods for simulations in constant- $\mathrm{pH}$ conditions because a classical description of protons is an issue. Instead, one models the effect of $\mathrm{pH}$ by fixing the protonation state of lipids and membrane proteins to the one that is consistent with a given $\mathrm{pH}$. Another important limitation is the length and timescale accessible to atomistic and coarse-grained MD simulations. The scales currently accessible to atomistic simulations are about $20 \mathrm{~nm} \times 20$ $\mathrm{nm} \times 20 \mathrm{~nm}$ and $1 \mu \mathrm{s}$. In CG descriptions, the scales depend on the level of coarse graining, that is, how much one has simplified the underlying atomistic representation, but some flavor of typical scales is gained by assuming that if the system size is the same as in atomistic models, then the timescale can usually be multiplied by a factor of $10^{3}$ to $10^{6}$ (Marrink et al. 2004; Murtola 2009; Ayton and Voth 2009). If these scales sound small, it is worthwhile to recall that only 10 years ago simulations of small lipid bilayers covered about $10 \mathrm{~ns}$, and protein simulations only about $1 \mathrm{~ns}$. The progress in biomolecular simulations has been truly impressive.

Nonetheless, to speed up atomistic lipid simulations even further, an obvious way is to design more efficient algorithms and their implementations. Particularly considerable progress has been made recently in the development and implementation of efficient schemes for parallel simulations. In this manner, the efficiency of the commonly used simulation engines such as GROMACS (Hess et al. 2008) and NAMD (Phillips et al. 2005) has been improved significantly, and currently both of these simulation packages allow one to use 500 to 1000 processors efficiently. Another 
possibility is to develop algorithms that are computationally less expensive but still of high accuracy. A good example in this context is the Particle Mesh Ewald technique (Essman et al. 1995), which computes electrostatic interactions much faster than the original formulation based on the Ewald summation scheme.

In the field of coarse graining, the progress during the last decade has been quite impressive. For given CG representations of molecules, several techniques to determine the effective interactions have been developed. Two oftenused schemes are based on matching either forces (Yanting et al. 2009) or structural properties (Murtola et al. 2009) between the coarsegrained and the underlying atomistic systems. An alternative technique is to consider the thermodynamic free energy for partitioning in a water-like or oil-like environment, and to parameterize the coarse-grained model in this spirit. The MARTINI model (Marrink et al. 2004, 2007; Monticelli et al. 2008; Lopez et al. 2009), using this idea, has turned out to be very successful in lipid simulations.

\section{LIPIDS IN ACTION}

Lipids Interacting with Peptides and Membrane Proteins

Membrane proteins constitute a large and important class of proteins. As about $30 \%$ of the genome codes for membrane proteins, and because studies of their dynamics are a challenge for any experimental technique, it is not surprising that $\mathrm{MD}$ simulations have become one of the most versatile techniques to explore their structure and dynamics (Kandt et al. 1998; Khalili-Araghi et al. 2009; Klepeis et al. 2009). Membrane proteins come in two varieties: peripheral proteins loosely associated with the membrane-water interface and integral proteins spanning the membrane. Aside from these two, there are also peptides associated with membranes.

Peptides provide an excellent opportunity to show how MD simulations complement experiments because the timescales related to peptide dynamics are much shorter compared to the dynamics of more complex proteins. Simulations of peptides provide a means to explore in atomistic detail the molecular phenomena that take place in 1-1000 nanoseconds. To this end, let us consider one of the biologically relevant functions of peptides: the formation of pores across lipid membranes (Fig. 2). When pores are formed, they facilitate the translocation of ions and small molecules through a membrane. When that takes place, cells go through lysis and die. Antimicrobial peptides use this mechanism to kill cells.

Peptides interacting with membranes are typically amphipatic, that is, composed of hydrophobic and hydrophilic (usually lysine and
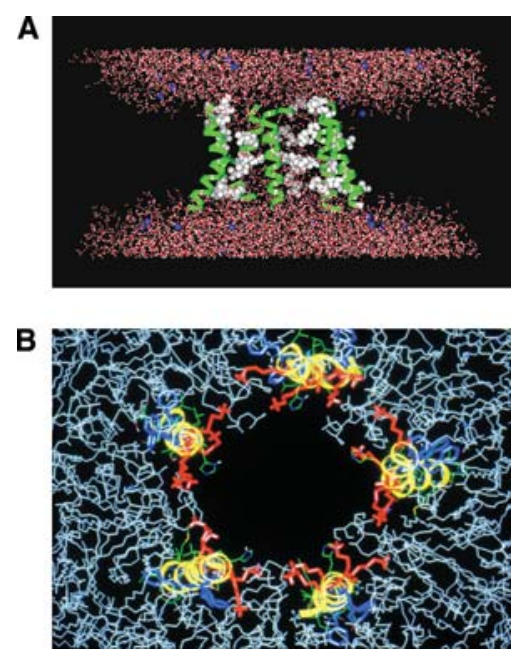

Figure 2. Upper panel: Side view of a magainin 2POPG pore in the lipid bilayer composed of a mixture of palmitoyloleoyl-phosphatidylglycerol (POPG) and phosphatidylethanolamine lipids. For clarity, lipids and hydrocarbon chains in the bilayer are not shown. Magainin molecules are depicted in green using the ribbon representation, and POPC head groups that are part of a pore are shown in white as van der Waals spheres. $\mathrm{Na}^{+}$ions are shown in blue. (Panel adapted from Pasenkiewicz-Gierula et al. [2000] and reprinted with permission from the author.) Lower panel: Top view of the pore. For clarity, water molecules are not shown. Lipids are represented as white lines, whereas magainin is shown as a yellow ribbon with blue hydrophobic side-chains and red lysine side-chains. (Panel adapted from Murzyn et al. [2004] and reprinted here with permission from Springer (C) 2004.) 
arginine) amino acids. A good example is magainin 2, an antimicrobial peptide originating from the skin of an African frog Xenopus laevis (Cruciani et al. 1992). The helical structure of magainin is characterized by its apolar residues facing the membrane, whereas the polar residues are exposed to water. The cooperative selfassembly of five to six magainin molecules leads to the formation of a toroidal membranespanning pore. The first computational model of the pore constructed nearly 10 years ago showed that the peptide-induced pore complex stabilized itself in nanoseconds (PasenkiewiczGierula et al. 2000; Murzyn et al. 2004). The mechanism by which the pore is formed was shown in a more recent study (Leontiadou et al. 2006). It was found that pore formation is a comparatively rapid process, taking place in about 10-100 ns, provided that there are a sufficient number of peptides nearby. Pores were found to be clearly permeable for water and lipids, but they were also observed to be rather disordered, showing greater variability in terms of peptide conformation and orientation than it was believed earlier. Similar studies were recently performed on another antimicrobial peptide, melittin, which also showed the disordered nature of pore formation (Sengupta et al. 2008). Overall, simulations have been able to shed light on the complex mechanisms by which pores form and function, providing atomistic and molecular-scale insight that is inaccessible to experiments.

Meanwhile, MD simulations of membrane proteins are computationally much more challenging compared to peptide simulations. This is largely because of the larger scales in time and space needed to simulate membrane proteins. Typical system sizes and timescales that are currently feasible are about 1,000,000 atoms simulated over a millisecond (Bjelkmar et al. 2009; Khalili-Araghi et al. 2009; Klepeis et al. 2009). The most commonly studied proteins are membrane channels including aquaporins, ion channels, and mechanosensitive channels. Simulations of these channels provide detailed information about the mechanisms associated with their conductance. For aquaporins, MD simulations were able to show a collective transport mechanism by which up to ten water molecules migrated through the channel in a concerted fashion; the dynamics of protein side chains played an important role in this process (Jensen et al. 2008). For the $\mathrm{Na}^{+} / \mathrm{H}^{+}$ antiporter, MD simulations showed a detailed patch of $\mathrm{Na}^{+}$ions and identified the key residues inside the channel that are responsible for transport (Arkin et al. 2007). These findings were confirmed in mutagenesis studies. A similar detailed picture has very recently been obtained for some of the $\mathrm{K}^{+}$(Jensen et al. 2010) and mechanosensitive channels (Vásquez et al. 2008).

Although the membrane in the abovementioned studies served only as an environment for the protein to carry out its function, it is known that lipids play an active role in protein functioning, which in turn is associated with specific lipid-protein interactions. This is highlighted by a recent simulation of rhodopsin in a three-component lipid bilayer composed of phosphatidylcholines, phosphatidylethanolamines, and cholesterols (Khelashvili et al. 2009). The simulation showed the distribution of cholesterol around rhodopsin to be nonuniform. Cholesterol was located next to the highly conserved GPCR family motive of the protein, stabilizing a specific conformation in this region (Khelashvili et al. 2009). In the same spirit for the Kv1.2 ion channel, one has found preferential binding of negatively charged phosphatidylglycerols (PGs): This enrichment of PGs was driven by interactions with the positively charged residues at the protein surface (Bjelkmar et al. 2009).

In the future, simulations are expected to provide further benefit for membrane science both in terms of unraveling phenomena associated with more complex proteins and in terms of considering more complex lipid compositions of membranes.

\section{Lipids and Proteins in Pharmacology}

When we are sick, a medical doctor makes a diagnosis and provides us with a prescription, and often the sickness is cured by the drugs we have been ordered. 
Does the success in treating the sickness also imply that we understand the action mechanism of the drug? No. In many cases it is not known, for example, how the drug binds to other molecules thus inhibiting their function and causing (for instance) inflammation to recover. What atomistic simulations can do is provide insight into the phenomena associated with the actions of drugs. Here we discuss some possibilities in this context.

When drugs act on biological membranes, the existing drugs have usually been targeted to affect membrane proteins. However, this practice is changing. Recently, as the role of lipids in drug-induced effects has been noticed, more attention has been paid to lipids as significant targets of drugs (Lúcio et al. 2010). A good example of this progress is lipid therapy, in which a drug molecule acts on a membrane to change the lipids' structure. In this way, they activate membrane receptors that in turn initiate a signaling pathway (Escriba 2006). Interestingly, the idea of lipid therapy is similar in spirit to the recent idea discussed by Cantor for the mechanism of general anesthetics (Cantor 1997; Jerabek et al. 2010). He proposed that anesthetic molecules could play a role in the activation of membrane proteins by changing the pressure profile of a lipid membrane. In this manner, lipids mediate interactions between anesthetics and proteins, as the force exerted on a protein by the membrane depends on the membrane composition. Another reason for the interest in drug-lipid interactions comes from the design of novel drug delivery systems based on lipids, such as liposomes and other lipid assemblies (Paasonen et al. 2007).

Computational methods have been used in drug design for a long time (Song et al. 2009). The most popular techniques have been structure-activity relationship and quantitative structure-activity relationship (Scior et al. 2009), as well as various docking methods based on scanning the protein surface and its cavities to find an optimal fit for the binding of a drug to the protein (Taylor et al. 2002). Currently, atomistic molecular dynamics simulations are not typically used in drug design because of the high computational cost. This is unfortunate because they can provide substantial insight into molecular mechanisms associated with the action of a drug and provide information about the interactions of a drug with other important biomolecules such as lipids.

To show the significant benefit of atomistic simulations, let us consider the MD simulation of $\beta 2$-adrenergic receptor ( $\beta 2$-AR) with nebivolol (Fig. 3) (Kaszuba et al. 2010). $\beta$-ARs belong to the family of G-protein coupled receptors (GPCRs) that constitute about $80 \%$ of the known receptors for neurotransmitters, hormones, and neuromodulators, and about $5 \%$ of the genes in eukaryotic organisms (Vigh et al. 2005). A list of diseases related to GPCR is long and includes, among others, certain forms of blindness, obesity, inflammation, depression, cancer, and hypertension (Lúcio et al. 2010). Therefore, not surprisingly, these receptors are one of the main targets in pharmacology. $\beta 2$-AR activity induces smooth muscle relaxation; thus, $\beta 2$-AR blockade is commonly used in asthma inhalers (Taylor 2007). Meanwhile, nebivolol is a new, highly selective $\beta 1$ AR blocker (Prisant et al. 2008) characterized by lack of side effects that are otherwise typical for $\beta$-blockers, and also lack of interactions with other drugs (Veverka et al. 2006). Nebivolol has four chiral centers and 10 stereoisomers, though only two of them are of pharmaceutical interest: the srrr-form and the rsss-form (Siebert et al. 2008).

Very recently, atomistic molecular dynamics simulations were used to unravel the issue of nebivolol selectivity toward one of the receptor's subtypes, as well as to understand its stereo-specificity (Kaszuba et al. 2010). Simulations clearly showed that the srrr-form interacts more favorably with the receptor, demonstrating the capacity of atomistic MD simulations for determining stereo-specificity. Most interestingly, this effect was observed to arise from energetically favorable interactions with water molecules. This finding is profoundly interesting because it highlights the important role of hydration of the bonding pocket and, more generally, the importance of water in drugprotein interactions. This perspective is of 
A

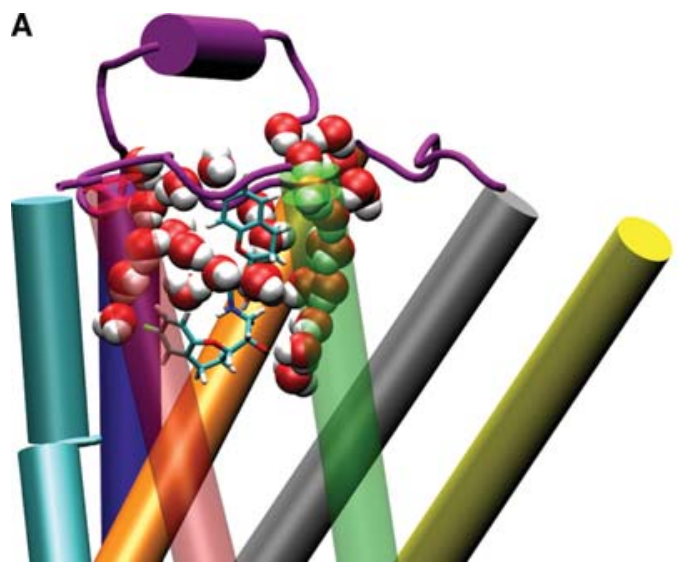

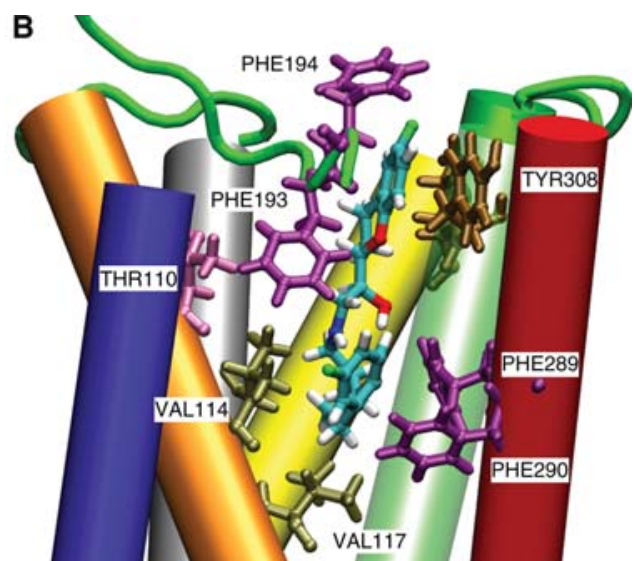

Figure 3. View of the binding pocket of $\beta 2$-AR occupied by a nebivolol molecule (Kaszuba et al. 2010). The left panel shows water molecules present in the pocket, whereas the right panel shows protein residues involved in direct interaction with nebivolol.

general importance for drug design because most of the docking methods commonly used in computational studies of drug-protein complexes do not include water. Clearly, there is reason to revise the docking techniques to account for this aspect.

\section{Lipoproteins as Carriers of Cholesterol}

Cardiovascular diseases (CVD) are the primary cause of death in western countries (Mokhad et al. 2004), causing about one out of five deaths in the population. One of the main causes of this condition is atherosclerosis, that is, the lipid accumulation and plaque formation on arterial walls. This severe condition relates to lipoprotein particles that transport cholesterol in the body. High levels of low-density lipoprotein (LDL) particles in blood have been found to increase the risk of atherosclerosis (Castelli et al. 1986; Hevonoja et al. 2000), whereas high levels of high-density lipoprotein (HDL) are known to reduce the risk (Colvin and Parks 1999; Linsel-Nitschke and Tall 2005).

To understand the functions of HDL and LDL, one should first know their structures, but despite extensive studies, this issue has remained unclear. A number of different models have been proposed for HDL (Phillips et al. 1997; Segrest et al. 1999; Martin et al. 2006; Wu et al. 2009) and LDL (Hevonoja et al. 2000), but a large amount of uncertainty has remained because the nanoscale size of HDL and LDL and the thermally fluctuating nature of these nanoparticles have challenged the resolution of experimental approaches.

Meanwhile, the role of computer simulations in lipoprotein research has increased steadily, and a number of simulation studies about lipoproteins have been published recently. For example, the properties of the lipoprotein core have been studied both for cholesteryl esters and triglycerides (Heikelä et al. 2006; Hall et al. 2008). HDL discs have also been paid considerable attention (Shih et al. 2005, 2008; Catte et al. 2006), and recent work on spheroidal HDL has been very illuminating (Catte et al. 2008; Koivuniemi et al. 2009). Especially, HDL simulations have been able to provide a great deal of insight into the atomistic structure of both the lipid droplet and the apolipoproteins ApoA-I surrounding the lipid part of HDL, and the important role of free cholesterol in stabilizing ApoA-I structure at the surface of HDL has become apparent.

A recent study (Yetukuri et al. 2010) shows the benefit of bridging molecular simulations to experiments particularly well. In that article, the authors considered two subject groups whose living habits were very different. One of 
the groups favoring a healthy diet and good living habits had a high level of HDL, whereas the other group with less healthy living habits had low HDL. High-throughput mass spectroscopy used to analyze the HDL lipid compositions revealed major differences, especially in the molar fractions of free cholesterol and triglycerides, illustrating that people with low HDL had reduced amounts of free cholesterol and larger amounts of triglycerides. The analyzed lipid compositions were employed in CG molecular simulations to compare the HDL structures of subjects in the two different groups. The results found via simulations were quite fascinating (Fig. 4): In low-HDL subjects, triglycerides, instead of being confined to the hydrophobic core of HDL, were observed to migrate to the HDL-water interface in which their concentration was markedly larger compared to the highHDL case. At the surface region, the elevated triglyceride concentration was coupled to a reduced concentration of free cholesterol, implying that the likelihood of triglyceride-ApoA-I contacts increased at the expense of a reducing number of contacts between free cholesterol and ApoA-I. As ApoA-I has been found to prefer contacts with cholesterol, the excess triglyceride replacing cholesterol in low-HDL subjects has likely a role to play in the function of HDL.

Although more extensive studies are needed, it seems plausible that diet and living habits influence the structure of the lipid droplet in HDL. That, in turn, may have an effect on the structure of ApoA-I and, hence, the functions of HDL. In a more general context, the study (Yetukuri et al. 2010) illustrates the benefit of coupling clinical research to bioinformatics and systems biology, and further to molecular simulations.
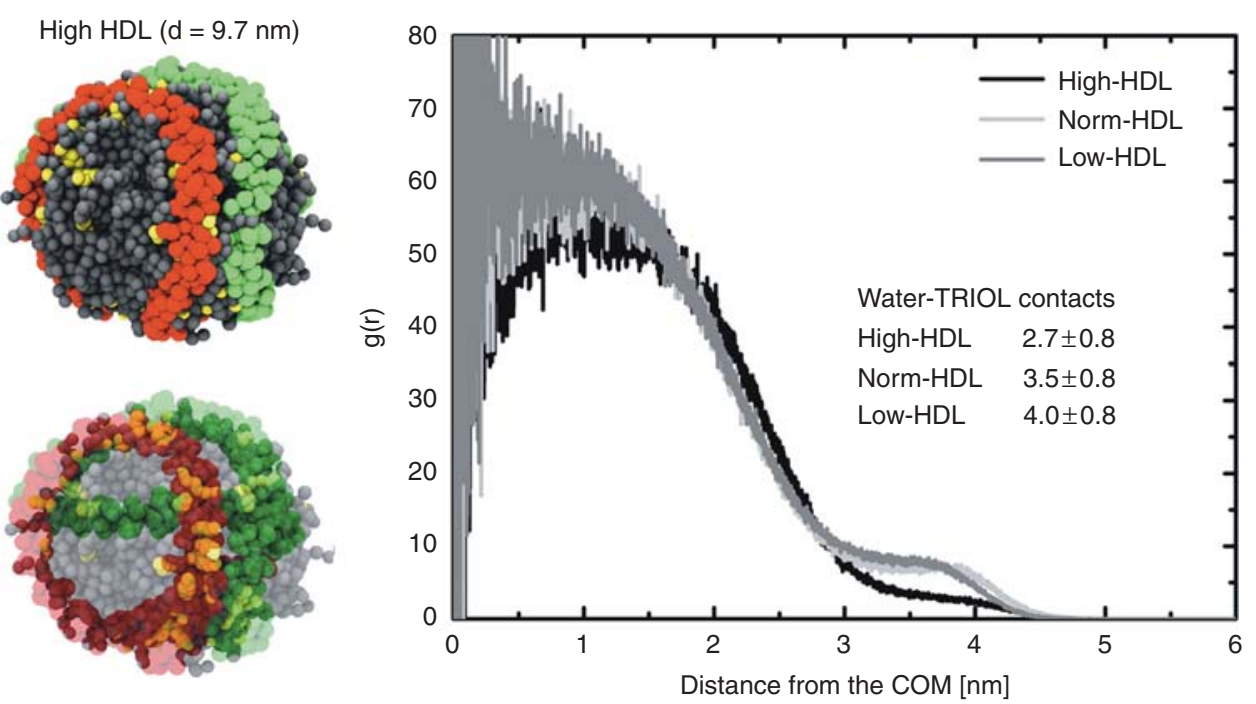

Figure 4. Coarse-grained simulation results of HDL particles reconstituted based on lipidomics data. Left: snapshots from high-HDL simulations. ApoA-Is are colored with red and green, cholesterol molecules are yellow, and all other lipids gray. Water phase was removed from the snapshots for clarity. Right: radial distribution function for triglyceride molecules, $g(r)$, with respect to the center-of-mass of HDL particle. The number of contacts between triglycerides and water beads in simulations for high, normal, and low HDL levels is shown in the inset. (Figure adapted from Koivuniemi et al. [2010] and reprinted with permission from Elsevier (C) 2010.) 


\section{Complex Concerted Dynamics in Membranes}

The lipid raft model (Simons and Ikonen 1997) and the extensive research that has followed this proposition have provided a great deal of insight into the structural aspects of biological membranes. Meanwhile, the understanding of membrane properties is largely incomplete, as rather little is known of the dynamics associated with membrane domains, their formation through diffusion of lipids, and even the diffusion of lipids in model membranes.

Cell membranes bustle with dynamic phenomena at a wide range of characteristic timescales and length scales. Motion of lipids in the plane of the membrane-lateral diffusion-is the most widely studied of these, and yet the mechanism of lateral diffusion has been far from well understood. It has been thought rather commonly that lateral diffusion follows the jump-diffusion model in which diffusion consists of rapid "rattling" motion of lipids confined to cages formed by their neighbors, punctuated by nearly instantaneous, discrete jumps in which a whole lipid molecule moves out of its cage, moving a distance comparable to its size. Short-range techniques, such as quasielastic neutron scattering (QENS), have been thought to measure the rapid rattling-in-a-cage motion, whereas long-range techniques, such as fluorescence recovery after photobleaching, have been assumed to gauge the slower motion consisting of jumps between cages. Phenomenological free volume models based on the jump-diffusion model have been used to interpret the dependence of lateral diffusion coefficients on temperature and membrane composition (Galla et al. 1979; MacCarthy and Kozak 1982; O’Leary 1987; Almeida et al. 1992). However, no direct experimental evidence for the jumps as a dominating diffusion mechanism has been reported.

Recent atomistic and coarse-grained simulations have shed light on this issue (Falck et al. 2007; Apajalahti et al. 2010). Falck et al. considered single-component lipid bilayers and analyzed the trajectories of all lipids in detail. They did not find evidence for jump-like motion in which individual lipids would have rapidly moved a distance of their own size in the bilayer plane in a slowly changing environment. Instead, Falck et al. identified the motion of transient lipid clusters in which about ten lipids moved in unison as loosely defined clusters (Fig. 5). Over larger scales, they further observed transient dynamic correlations in which the motions of lipids were coupled to one another over scales of $\sim 10 \mathrm{~nm}$. In a more recent work (Apajalahti et al. 2010), coarsegrained simulations were used to consider similar phenomena in raft-like membranes over much larger scales in time and space. They observed the dynamical correlations in lateral motion to persist over a microsecond timescale. Recent simulation studies (Roark and Feller 2009) have also provided support to the concerted diffusion mechanism.

Importantly, the simulations by Falck et al. predicted that the lipid diffusion in membranes takes place through concerted motions of lipids as transient lipid clusters. Experimental data of these phenomena did not exist until early 2010 (Busch et al. 2010), when the Unruh group reported QENS data for lipid diffusion. Their data confirmed the predictions of simulations, highlighting the concerted motion of lipids as the mechanism of lipid diffusion.

Flow-like patterns have earlier been found in hydrodynamic two-dimensional systems in which the driving force has been the conservation of momentum. In lipid diffusion, momentum is not conserved within a membrane because lipids exchange momentum with the water phase. The origin of the dynamical correlations observed in membranes is therefore different: There are local and transient density waves that emerge spontaneously, giving rise to the observed large-scale motions. The findings highlight the importance of spontaneous collective fluctuations and suggest that similar phenomena are abundant also in other soft matter systems. Most recently, this has been observed for integral membrane proteins whose motion also takes place in a concerted manner with 50 to 100 lipids around it (Niemela et al. 
I. Vattulainen and T. Rog
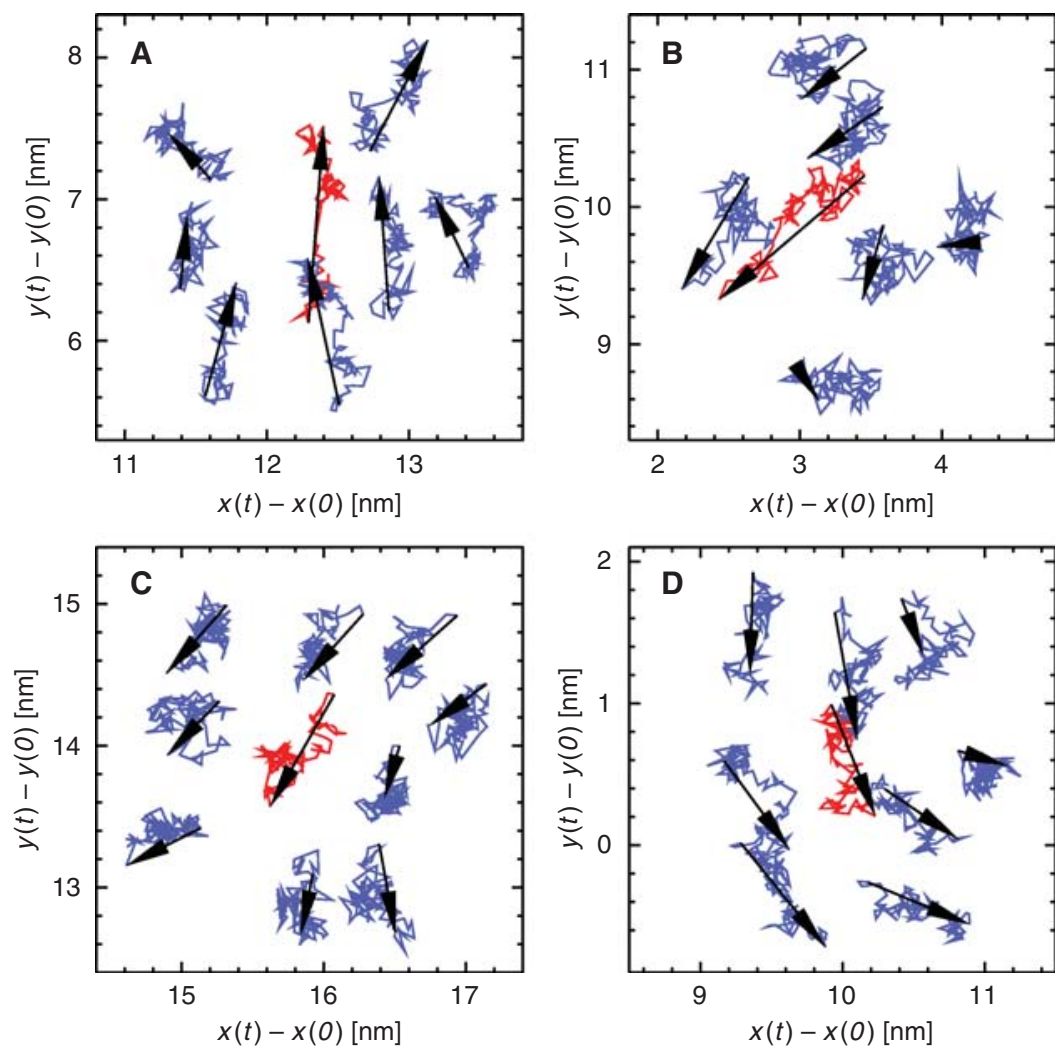

Figure 5. Trajectories of neighboring lipids. The trajectory in the middle of each panel (shown in red) describes the motion of a lipid undergoing the actual diffusion event in which the lipid migrates the longest distance during a $1 \mathrm{~ns}$ time interval. The other trajectories in each panel are those of the nearest neighbors, that is, the lipids whose center-of-mass are within $1 \mathrm{~nm}$ of that of the central lipid in panels $A, B, C$, or $D$ at any time during the 1-ns interval. The arrows indicate the total displacements during the 1-ns period. (Figure adapted from Falck et al. [2007] and reprinted with permission from American Chemical Society (C) 2007.)

2010). This finding may have considerable significance to the dynamics and function of cell membranes, as it implies that the dynamics of proteins and lipids in cell membranes are not two separate issues but have to be considered together.

\section{CONCLUDING REMARKS}

Computer simulations of lipid systems have been performed since the 1980s. During these decades, the role of lipid simulations has increased significantly. Although in the early times simulations mainly confirmed what had been seen in experiments, the field of lipid simulations has now matured to a level in which it can also guide experiments by making predictions of novel phenomena not yet observed in experimental laboratories. For example, the concerted motions of diffusing molecules in lipid bilayers were first observed in atomistic simulations (Falck et al. 2007), and later confirmed in QENS experiments (Busch et al. 2010). Membrane protein structures can be resolved by crystallographic means, but the dynamics of the proteins can be investigated by computer simulations, providing a way to predict and clarify their activation mechanisms (Bjelkmar 2009). Simulations can also generate insight into phenomena that cannot be studied 
experimentally, such as in quantifying the magnitude and range of membrane perturbations induced by fluorescent lipid probes (Repakova 2005; Hölttävuori 2008). Considering the benefit of bridging experiments with simulations, we are confident that this trend will be promoted, fostering better science.

\section{ACKNOWLEDGMENTS}

Artturi Koivuniemi, Emma Falck, and Karol Kaszuba are thanked for graphical presentations. The Academy of Finland is acknowledged for financial support. CSC - IT Centre for Science (Finland) as well as the HorseShoe computing cluster in the University of Southern Denmark are thanked for computational resources.

\section{REFERENCES}

Almeida PFF, Vaz WLC, Thompson TE. 1992. Lateral diffusion in the liquid phases of dimyristoylphosphatidylcholine/cholesterol lipid bilayers: A free volume analysis. Biochemistry 31: 6739-6747.

Apajalahti T, Niemela P, Govindan PN, Miettinen MS, Salonen E, Marrink SJ, Vattulainen I. 2010. Concerted diffusion of lipids in raft-like membranes. Faraday Discuss 144: $411-430$.

Arkin IT, Xu H, Jensen MØ, Arbely E, Bennett ER, Bowers KJ, Chow E, Dror RO, Eastwood MP, Flitman-Tene R, et al. 2007. Mechanism of $\mathrm{Na}^{+} / \mathrm{H}^{+}$antiporting. Science 317: 799-803.

Ayton GS, Voth GA. 2009. Systematic multiscale simulation of membrane protein systems. Curr Opin Struct Biol 19: $138-144$.

Bjelkmar P, Niemela P, Vattulainen I, Lindahl E. 2009. Conformational changes and slow dynamics through microsecond polarized molecular simulation of an integral Kv1.2 ion channel. PLoS Comput Biol 5: e1000289.

Bucher D, Guidoni L, Carloni P, Rothlisberger U. 2010. Coordination numbers of $\mathrm{K}^{+}$and $\mathrm{Na}^{+}$ions inside the selectivity filter of the KcsA potassium channel: Insights from first principles molecular dynamics. Biophys $J$ 98: L47-L49.

Busch S, Smuda C, Pardo LC, Unruh T. 2010. Molecular mechanism of long-range diffusion in phospholipid membranes studied by quasielastic neutron scattering. J Am Chem Soc 132: 3232-3233.

Cantor SR. 1997. The influence of membrane lateral pressure on simple geometric models of protein conformational equilibria. Biochemistry 36: 2339-2344.

Castelli WP, Garrison RJ, Wilson PW, Abbott RD, Kalousdian S, Kannel WB. 1986. Incidence of coronary heart disease and lipoprotein cholesterol levels. The Framingham study. JAMA 256: 2835-2838.
Catte A, Patterson JC, Bashtovyy D, Jones MK, Gu F, Li L, Rampioni A, Sengupta D, Vuorela T, Niemela P, et al. 2008. Structure of spheroidal HDL particles revealed by combined atomistic and coarse grained simulations. Biophys J 94: 2306-2319.

Catte A, Patterson JC, Jones MK, Jerome WG, Bashtovyy D, Su Z, Gu F, Chen J, Aliste MP, Harvey SC, et al. 2006. Novel changes in discoidal high density lipoprotein morphology: A molecular dynamics study. Biophys $J$ 90: 4345-4360.

Colvin PL, Parks JS. 1999. Metabolism of high density lipoprotein subfractions. Curr Opin Lipidol 10: 309-314.

Cruciani RA, Barker JL, Durell SR, Raghunathan G, Guy HR, Zasloff M, Stanley EF. 1992. Magainin 2, a natural antibiotic from frog skin, forms ion channels in lipid bilayer membranes. Eur J Pharmacol 226: 287-296.

Dror RO, Arlow DH, Borhani DW, Jensen MO, Piana S, Shaw DE. 2009. Identification of two distinct inactive conformations of the $\beta_{2}$-adrenergic receptor reconciles structural and biochemical observations. Proc Natl Acad Sci 106: 4689-4694.

Escriba PV. 2006. Membrane-lipid therapy: A new approach in molecular medicine. Trends Mol Med 12: 34-43.

Essman U, Perera L, Berkowitz ML, Darden HLT, Pedersen LG. 1995. A smooth particle mesh Ewald method. J Chem Phys 103: 8577-8593.

Falck E, Rog T, Karttunen M, Vattulainen I. 2007. Lateral diffusion in lipid membranes through collective flows. J Am Chem Soc 130: 44-45.

Fan J, Sammalkorpi M, Haataja M. 2010a. Formation and regulation of lipid microdomains in cell membranes: Theory, modeling, and speculation. FEBS Lett 584: $1678-1684$.

Fan J, Sammalkorpi M, Haataja M. 2010b. Influence of nonequilibrium lipid transport, membrane compartmentalization, and membrane proteins on the lateral organization of the plasma membrane. Phys Rev E 81: 011908.

Frenkel D, Smit B. 2002. Understanding molecular simulation. 2nd ed. Academic Press, San Diego, CA.

Galla HJ, Hartmann W, Theilen U, Sackmann EJ. 1979. On two-dimensional passive random walk in lipid bilayers and fluid pathways in biomembranes. J Membr Biol 48: 215-236.

Hall A, Repakova J, Vattulainen I. 2008. Modelling of the triglyceride-rich core in lipid droplets. J Phys Chem B 112: $13772-13782$.

Heikelä M, Vattulainen I, Hyvönen MT. 2006. Atomistic simulation studies of cholesteryl oleates in the interior of lipoprotein particles. Biophys J 90: 2247-2257.

Hess B, Kutzner C, van der Spoel D, Lindahl E. 2008. GROMACS 4: Algorithms for highly efficient, loadbalanced, and scalable molecular simulation. J Chem Theory Comput 4: 435-447.

Hevonoja T, Pentikäinen MO, Hyvönen MT, Kovanen PT, Ala-Korpela M. 2000. Structure of low density lipoprotein (LDL) particles: Basis for understanding molecular changes in modified LDL. Biochim Biophys Acta 1488: $189-210$.

Hölttävuori M, Uronen RL, Repakova J, Salonen E, Vattulainen I, Panula P, Li Z, Bittman R, Ikonen E. 2008. BODIPY-cholesterol: A new tool to visualize sterol 
trafficking in living cells and organisms. Traffic 9: 1839-1849.

Jensen MØ, Borhani DW, Lindorff-Larsen K, Maragakis $\mathrm{P}$, Jogini V, Eastwood MP, Dror RO, Shaw DE. 2010. Principles of conduction and hydrophobic gating in $\mathrm{K}^{+}$channels. Proc Natl Acad Sci 107: 5833-5838.

Jensen MØ, Dror RO, Xu H, Borhani DW, Arkin IT, Eastwood MP, Shaw DE. 2008. Dynamic control of slow water transport by aquaporin 0 : Implications for hydration and junction stability in the eye lens. Proc Natl Acad Sci 105: 14430-14435.

Jerabek H, Pabst G, Rappolt M, Stockner T. 2010. Membrane-mediated effect on ion channels induced by the anesthetic drug ketamine. J Am Chem Soc 132: 7990-7997.

Kandt C, Mátyus E, Tieleman DP. 1998. Protein lipid interactions from a simulation point of view. In Structure and dynamics of membranous interfaces (ed. K. Nag), pp. 267-282. John Wiley \& Sons, Hoboken, NJ.

Kaszuba K, Róg T, Bryl K, Vattulainen I, Karttunen M. 2010. Molecular dynamics simulations reveal fundamental role of water as factor determining affinity of binding of $\beta$-blocker nebivolol to $\beta_{2}$-adrenergic receptor. J Phys Chem B 114: 8374-8386.

Khalili-Araghi F, Gumbart J, Wen PC, Sotomayor M, Tajkhorshid E, Schulten K. 2009. Molecular dynamics simulations of membrane channels and transporters. Curr Opin Struct Biol 19: 128-137.

Khelashvili G, Grossfield A, Feller SE, Pitman MC, Weinstein H. 2009. Structural and dynamic effects of cholesterol at preferred sites of interaction with rhodopsin identified from microsecond length molecular dynamics simulations. Proteins 76: 403-417.

Klepeis JL, Lindorff-Larsen K, Dror RO, Shaw DE. 2009. Long-time scale molecular dynamics simulations of protein structure and function. Curr Opin Struct Biol 19: $120-127$.

Koivuniemi A, Heikela M, Kovanen PT, Vattulainen I, Hyvonen MT. 2009. Atomistic simulations of phosphatidylcholines and cholesteryl esters in high-density lipoprotein-sized lipid droplet and trilayer: Clues to cholesteryl ester transport and storage. Biophys $J$ 96: 4099-4108.

Kox AJ, Michels JPJ, Wiegel FW. 1980. Simulation of a lipid monolayer using molecular dynamics. Nature 287: 317-319.

Leontiadou H, Mark AE, Marrink SJ. 2006. Antimicrobial peptides in action. J Am Chem Soc 128: 12156-12161.

Lingwood D, Simons K. 2010. Lipid rafts as a membraneorganizing principle. Science 327: 46-50.

Linsel-Nitschke P, Tall AR. 2005. HDL as a target in the treatment of atherosclerotic cardiovascular disease. Nat Rev Drug Discov 4: 193-205.

Lopez CA, Rzepiela A, de Vries AH, Dijkhuizen L, Huenenberger PH, Marrink SJ. 2009. The Martini coarse grained force field: Extension to carbohydrates. J Chem Theory Comput 5: 3195-3210.

Lúcio M, Lima JLFC, Reis S. 2010. Drug-membrane interactions: Significance for medicinal chemistry. Curr Med Chem 17: 1795-1809.
MacCarthy JE, Kozak JJ. 1982. Lateral diffusion in fluid systems. J Chem Phys 77: 2214-2216.

Marrink SJ, de Vries AH, Mark AE. 2004. Coarse grained model for semi-quantitative lipid simulations. J Phys Chem B 108: $750-760$.

Marrink SJ, Risselada HJ, Yefimov S, Tieleman DP, de Vries AH. 2007. The MARTINI forcefield: Coarse grained model for biomolecular simulations. J Phys Chem B 111: 7812-7824.

Martin DD, Budamagunta MS, Ryan RO, Voss JC, Oda MN. 2006. Apolipoprotein A-I assumes a "looped belt" conformation on reconstituted high density lipoprotein. J Biol Chem 281: 20418-20426.

McIntosh TJ, Simon SA. 2006. Roles of bilayer material properties in function and distribution of membrane proteins. Annu Rev Biophys Biomol Struct 35: 177-198.

Mokdad AH, Marks JS, Stroup DF, Gerberding JL. 2004. Actual causes of death in the United States, 2000 JAMA 291: 1238-1245.

Monticelli L, Kandasamy SK, Periole X, Larson RG, Tieleman DP, Marrink SJ. 2008. The MARTINI coarse grained force field: Extension to proteins. J Chem Theory Comput 4: 819-834.

Mouritsen OG. 2005. Life—As a matter of fat. Springer, Heidelberg, Germany.

Murtola T, Bunker A, Vattulainen I, Deserno M, Karttunen M. 2009. Multiscale modeling of emergent materials: Biological and soft matter. Phys Chem Chem Phys 11: 1869-1892.

Murzyn K, Róg T, Pasenkiewicz-Gierula M. 2004. Interactions of magainin-2 amide with membrane lipids. Lect Notes Comp Sci 3037: 325-331.

Niemela P, Miettinen MS, Monticelli L, Hammaren H, Bjelkmar P, Murtola T, Lindahl E, Vattulainen I. 2010. Membrane proteins diffuse as dynamic complexes with lipids. J Am Chem Soc 132: 7574-7575.

O'Leary TJ. 1987. Lateral diffusion of lipids in complex biological membranes. Proc Natl Acad Sci 84: 429-433.

Paasonen L, Romberg B, Storm G, Yliperttula M, Urtti A, Hennink WE. 2007. Temperature-sensitive poly(N-(2hydroxypropyl)methacrylamide mono/dilactate)-coated liposomes for triggered contents release. Bioconjugate Chem 18: 2131-2136.

Pasenkiewicz-Gierula M, Murzyn K, Róg T, Czaplewski C. 2000. Molecular dynamics simulation studies of lipid bilayer systems. Acta Biochim Pol 47: 601-611.

Phillips JC, Braun R, Wang W, Gumbart J, Tajkhorshid E, Villa E, Chipot C, Skeel RD, Kale L, Schulten K. 2005. Scalable molecular dynamics with NAMD. J Comput Chem 26: $1781-1802$

Phillips JC, Wriggers W, Li Z, Jonas A, Schulten K. 1997. Predicting the structure of apolipoprotein A-I in reconstituted high-density lipoprotein disks. Biophys $J$ 73: 2337-2346.

Praprotnik M, Site LD, Kremer K. 2007. Multiscale simulation of soft matter: From scale bridging to adaptive resolution. Ann Rev Phys Chem 59: 545-571.

Prisant J. 2008. Nebivolol: Pharmacologic profile of an ultraselective, vasodilatory $\beta$ (1)-blocker. Clin Pharmacol 48: 225-239. 
Repakova J, Morrow MR, McDonald MC, Capkova P, Holopainen J, Vattulainen I. 2005. Influence of DPH on the structure and dynamics of a DPPC bilayer. Biophys J 88: 3398-3410.

Reynwar BJ, Illya G, Harmandaris VA, Müller MM, Kremer K, Deserno M. 2007. Aggregation and vesiculation of membrane proteins by curvature-mediated interactions. Nature 447: 461-464.

Roark R, Feller SE. 2009. Molecular dynamics simulation study of correlated motions in phospholipid bilayer membranes. J Phys Chem B 113: 13229-13234.

Sackmann E. 1995. Biological membranes architecture and function. In Structure and dynamics of membranes: From cells to vesicles (ed. R Lipowsky, E Sackmann), pp. 1-65. Elsevier, Amsterdam, The Netherlands.

Scior T, Medina-Franco JL, Do QT, Martínez-Mayorga K, Yunes-Rojas JA, Bernard P. 2009. How to recognize and workaround pitfalls in QSAR studies: A critical review. Curr Med Chem 16: 4297-4313.

Segrest JP, Jones MK, Klon AE, Sheldahl CJ, Hellinger M, de Loof H, Harvey SC. 1999. A detailed molecular belt model for apolipoprotein A-I in discoidal high density lipoprotein. J Biol Chem 274: 31755-31758.

Sengupta D, Leontiadou H, Mark AE, Marrink SJ. 2008. Toroidal pores formed by antimicrobial peptides show significant disorder. Biochim Biophys Acta 1778: 23082317.

Senn HM, Thiel W. 2009. QM/MM methods for biomolecular systems. Angew Chem Intl Ed 48: 1198-1229.

Shih AY, Sligar SG, Schulten K. 2008. Molecular models need to be tested: The case of a solar flares discoidal HDL model. Biophys J 94: L87-L89.

Shih AY, Denisov IG, Phillips JC, Sligar SG, Schulten K. 2005. Molecular dynamics simulations of discoidal bilayers assembled from truncated human lipoproteins. Biophys J 88: 548-556.

Siebert CD, Hansicke A, Nagel T. 2008. Stereochemical comparison of nebivolol with other $\beta$-blockers. Chirality 20: 103-109.

Simons K, Ikonen E. 1997. Functional rafts in cell membranes. Nature 387: 569-572.

Singer SJ, Nicolson GL. 1972. The fluid mosaic model of the structure of cell membranes. Science 175: 720-731.

Song CM, Lim SJ, Tong JC. 2009. Recent advances in computer-aided drug design. Brief Bioinform 10: 579-591.
Taylor MRG. 2007. Pharmacogenetics of the humanadrenergic receptors. Pharmacogenomics J 7: 29-37.

Taylor RD, Jewsbury PJ, Essex JW. 2002. A review of proteinsmall molecule docking methods. J Comp Aided Mol Des 16: $151-166$.

Tieleman DP, Marrink SJ, Berendsen HJ. 1997. A computer perspective of membranes: Molecular dynamics studies of lipid bilayer systems. Biochim Biophys Acta 1331: 235-270.

van der Ploeg P, Berendsen HJC. 1982. Molecular dynamics simulation of a bilayer membrane. J Chem Phys 76: 3271-3276.

van der Ploeg P, Berendsen HJC. 1983. Molecular dynamics of a bilayer membrane. Mol Phys 49: 233-248.

van Gunsteren WF, Bakowies D, Baron R, Chandrasekhar I, Christen M, Daura X, Gee P, Geerke DP, Glattli A, Hunenberger PH, et al. 2006. Biomolecular modeling: Goals, problems, perspectives. Angew Chem Int Ed 45: 4064-4092.

van Meer G. 2005. Cellular lipidomics. EMBO J 24: 3159 3165.

Vásquez V, Sotomayor M, Cordero-Morales J, Schulten K, Perozo E. 2008. A structural mechanism for MscS gating in lipid bilayers. Science 321: 1210-1214.

Veverka A, Nuzum DS, Jolly JL. 2006. Nebivolol: A third generation $\beta$-adrenergic blocker. Ann Pharmacother 40: $1353-1360$.

Vigh L, Escriba PV, Sonnleitner A, Sonnleitner M, Piotto S, Maresca B, Horvath I, Harwood JL. 2005. The significance of lipid composition for membrane activity: New concepts and ways of assessing function. Prog Lipid Res 44: 303-344.

Wu ZP, Gogonea V, Lee X, Wagner MA, Li XM, Huang Y, Undurti A, May RP, Haertlein M, Moulin M, et al. 2009. The double super helix model of high density lipoprotein. J Biol Chem 284: 36605-36619.

Yanting W, Noid WG, Liu P, Voth GA. 2009. Effective force coarse-graining. Phys Chem Chem Phys 11: 2002-2015.

Yetukuri L, Soderlund S, Koivuniemi A, Seppanen-Laakso T, Niemela PS, Hyvonen M, Taskinen MR, Vattulainen I, Jauhiainen M, Oresic M. 2010. Lipid composition, structure and function of HDL particles in subjects with low and high HDL-cholesterol. J Lipid Res 51: 2341-2351. 


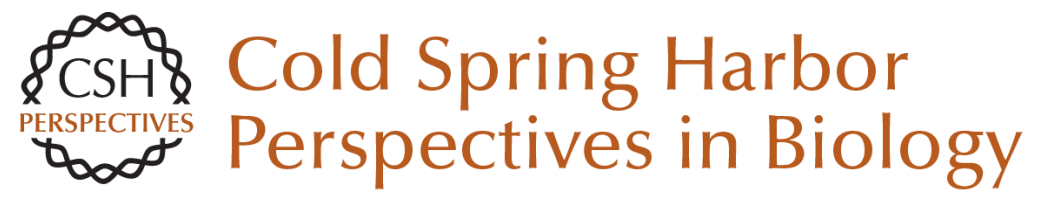

\section{Lipid Simulations: A Perspective on Lipids in Action}

Ilpo Vattulainen and Tomasz Rog

Cold Spring Harb Perspect Biol 2011; doi: 10.1101/cshperspect.a004655 originally published online February 9, 2011

Subject Collection The Biology of Lipids

Role of Lipids in Virus Replication Maier Lorizate and Hans-Georg Kräusslich

Model Answers to Lipid Membrane Questions Ole G. Mouritsen

\section{Glycosphingolipid Functions} Clifford A. Lingwood

Regulation of Cholesterol and Fatty Acid Synthesis

Jin Ye and Russell A. DeBose-Boyd

\section{Lipid-Mediated Endocytosis}

Helge Ewers and Ari Helenius

\section{Fluorescence Techniques to Study Lipid}

Dynamics

Erdinc Sezgin and Petra Schwille

Lysosomal Lipid Storage Diseases

Heike Schulze and Konrad Sandhoff

\section{Distribution and Functions of Sterols and} Sphingolipids

J. Thomas Hannich, Kyohei Umebayashi and Howard Riezman
Membrane Organization and Lipid Rafts Kai Simons and Julio L. Sampaio

Shotgun Lipidomics on High Resolution Mass

Spectrometers

Dominik Schwudke, Kai Schuhmann, Ronny

Herzog, et al.

Glycosphingolipid Functions Clifford A. Lingwood

Phosphoinositides in Cell Architecture Annette Shewan, Dennis J. Eastburn and Keith Mostov

Synthesis and Biosynthetic Trafficking of Membrane Lipids Tomas Blom, Pentti Somerharju and Elina Ikonen

Lipid Polymorphisms and Membrane Shape Vadim A. Frolov, Anna V. Shnyrova and Joshua Zimmerberg

Specificity of Intramembrane Protein-Lipid Interactions

Francesc-Xabier Contreras, Andreas Max Ernst, Felix Wieland, et al.

Dynamic Transbilayer Lipid Asymmetry Gerrit van Meer

For additional articles in this collection, see http://cshperspectives.cshlp.org/cgi/collection/

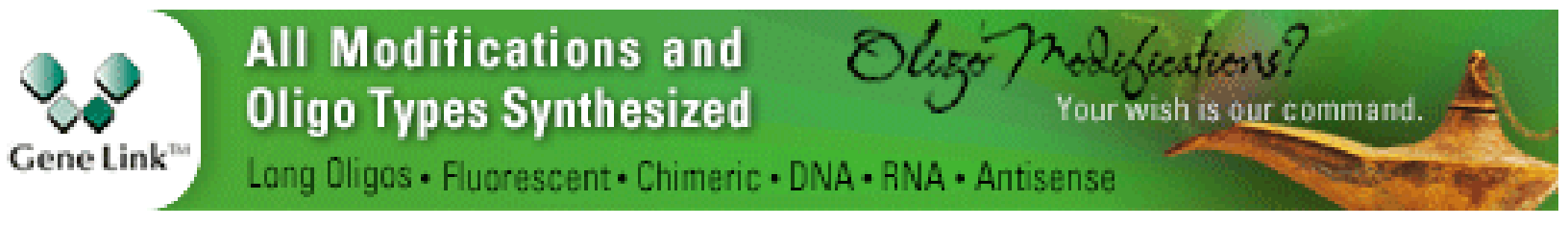

Copyright @ 2011 Cold Spring Harbor Laboratory Press; all rights reserved 